\title{
ELECTRON PARAMAGNETIC RESONANCE (EPR) SPECTRAL COMPONENTS OF SPIN-LABELED LIPIDS IN SATURATED PHOSPHOLIPID BILAYERS. EFFECT OF CHOLESTEROL
}

\author{
Heverton Silva Camargos ${ }^{\#}$ and Antonio Alonso* \\ Instituto de Física, Universidade Federal de Goiás, 74001-970 Goiânia - GO, Brasil
}

Recebido em 23/9/12; aceito em 7/1/13; publicado na web em 6/5/13

\begin{abstract}
Electron paramagnetic resonance (EPR) spectroscopy was used to study the main structural accommodations of spin labels in bilayers of saturated phosphatidylcholines with acyl chain lengths ranging from 16 to 22 carbon atoms. EPR spectra allowed the identification of two distinct spectral components in thermodynamic equilibrium at temperatures below and above the main phase transition. An accurate analysis of EPR spectra, using two fitting programs, enabled determination of the thermodynamic profile for these major probe accommodations. Focusing the analysis on two-component EPR spectra of a spin-labeled lipid, the influence of $40 \mathrm{~mol} \%$ cholesterol in DPPC was studied.
\end{abstract}

Keywords: EPR; spin label; lipid dynamics.

\section{INTRODUCTION}

For several decades, Electron Paramagnetic Resonance (EPR) spectroscopy of spin probes has contributed to a better understanding of cell membranes, particularly with regard to their dynamic and structural properties. However, the EPR spectra of lipid spin probes in biomembranes are very often quite complex and usually have more than one resolved or non-resolved spectral component. For instance, EPR spectra of two components are generally observed in the presence of integral membrane proteins, which is indicative of the coexistence of two classes of spin labels differing in their state of mobility. The more restricted component is associated with the boundary lipids where the spin labels surround the hydrophobic regions of integral proteins, while the more mobile component arises from the spin labels located in the bulk bilayer phase away from the protein. ${ }^{1,2} \mathrm{In}$ addition, two-component EPR spectra are also commonly observed for spin labels in simple bilayers and, since the dynamic properties are related to membrane permeability and function, it is an issue that warrants further study.

Studies of bilayer systems using fluorescence spectroscopy have also shown that several membrane probes may occupy at least two locations in the bilayer characterized by distinct polarity and hydration. ${ }^{3-7}$ The origin of this bimodal distribution has been interpreted based on the ability of these probes to participate in intermolecular hydrogen bonding within the membrane - a polar site at the bilayer interface where the probe is $\mathrm{H}$-bonded to water and an apolar site with no H-bonding to water. ${ }^{5}$ Interestingly, hydrostatic pressure applied on multilamellar vesicles of DMPC causes the relocation of the fluorescent probe Prodan towards more hydrophobic environments in the membrane. ${ }^{6}$ In previous studies, two-component EPR spectra of several spin labels in membranes of stratum corneum, the upper skin layer, were examined using the fitting program NLLS to monitor the dynamic and fraction of probe in each component. ${ }^{8-10}$ The presence of the terpenes L-menthol and 1,8-cineole, two potent skin permeation enhancers, in stratum corneum at $1 \%(\mathrm{w} / \mathrm{w})$ or DPPC vesicles at a terpene:lipid molar ratio of $0.7: 1$ drastically increased the lipid fluidity, especially by transferring the spin probes from a

*e-mail: alonso@if.ufg.br

\#Alternative address: Engenharia Elétrica, Fundação Universidade Federal do Tocantins, 77001 - 090 Palmas - TO, Brasil more to a less motionally restricted spectral component in the membranes. ${ }^{9,10}$ More recently, EPR spectra of spin labels in membrane were simulated using two-component models to study effects of the anti antileishmanial drug miltefosine in stratum corneum intercellular membranes ${ }^{11}$ and trypanocidal action of elatol ${ }^{12}$ as well as the toxicity of terpenes in cultured fibroblasts. ${ }^{13}$

Since the question of the spectral components in the EPR spectra of spin-labeled lipids in membrane models of simple bilayers has not been addressed in depth, we conducted an extensive experimental study with the spin probe methyl 5-doxyl-stearate (5-DMS) in vesicles of saturated phosphatidylcholines with acyl chain lengths ranging from 16 to 22 carbon atoms and temperatures in the 10 to 78 ${ }^{\circ} \mathrm{C}$ range. To gain a better understanding of the molecular interactions of this spin label within the membrane, the molecular dynamics of these two major structural accommodations and the thermodynamic profile involved in the probe distribution in these environments were determined for each membrane model studied. Using the same magnetic parameters set optimized for a simulation model with one or two components, we analyzed the influence of the addition of 40 mol \% cholesterol to the DPPC membrane on the mobility and spin-label population in each of these locations in the vesicle bilayers.

\section{EXPERIMENTAL}

\section{Chemicals}

The phospholipids 1,2-X-sn-glycero-3-phosphocholine, where $\mathrm{X}$ represents dipalmitoyl (DPPC), and distearoyl (PC-18), diarachidoyl (PC-20), dibehenoyl (PC-22), as well as the spin labels 1-palmitoyl-2-stearoyl-(5-doxyl)-sn-glycero-3-phosphocholine (5-PC), 10- and 16PC were purchased from Avanti (Avanti Polar Lipids, Inc., Alabaster, AL). The cholesterol and the spin labels methyl 5-, 12- and 16-doxyl-stearate (5-, 12- and 16-DMS) and 5-, 12- and 16-doxyl stearic acid (5-, 12- and 16-DSA) were purchased from Sigma (Chem. Co., St. Louis, MO). The molecular formulae of these amphiphilic molecules are shown in Figure 1.

\section{Preparation of vesicles}

Each sample was prepared with $5 \mathrm{mg}$ of phospholipid and spin label at a lipid:probe molecular ratio of (150:1), initially dissolved 


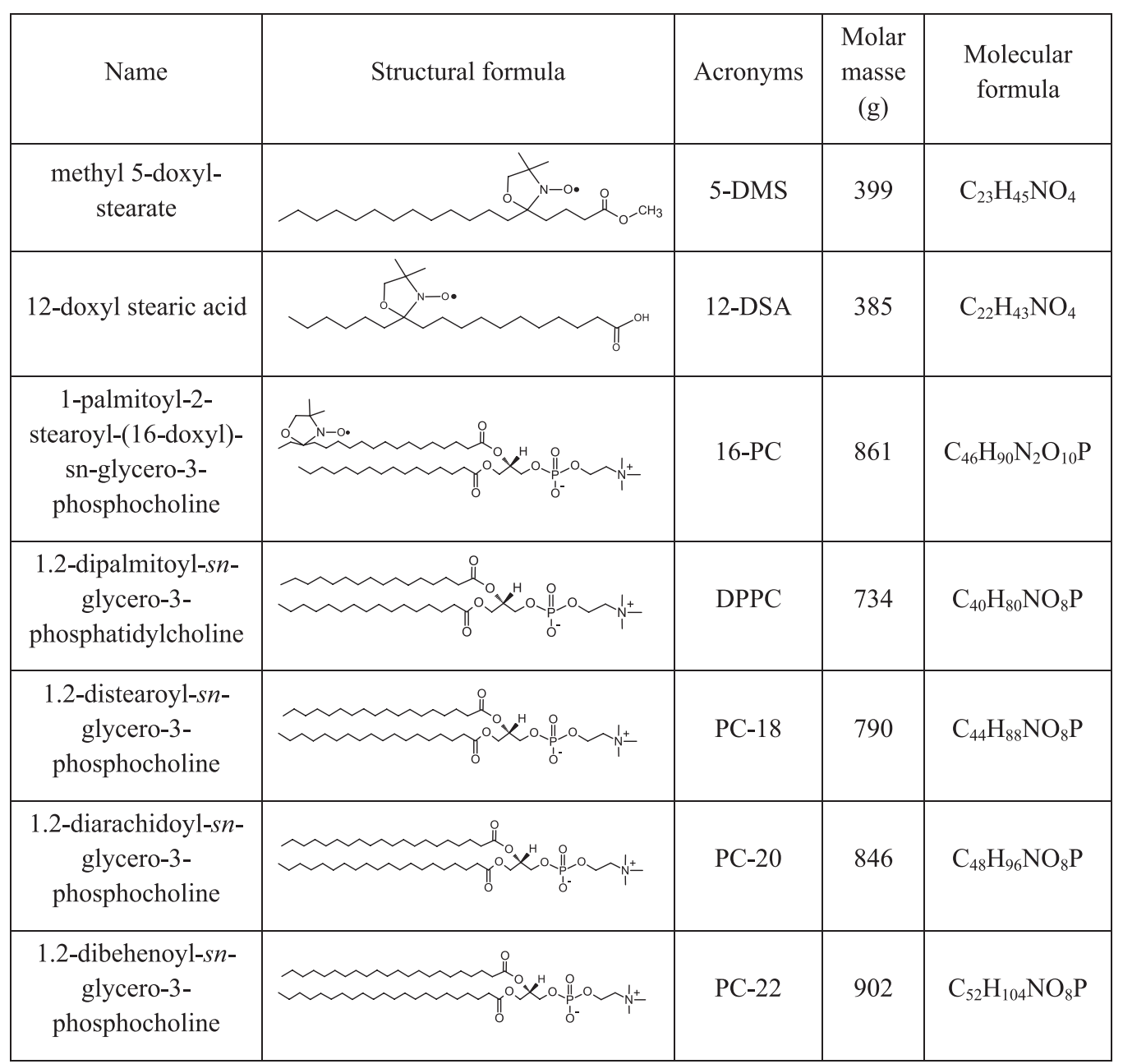

Figure 1. The structural and molecular formulae, molar masses, full names and acronyms of the amphiphilic molecules used in this work

in chloroform at a ratio of $5 \mathrm{mg} / \mathrm{mL}$. The organic phase was dripped into Milli-Q water at temperatures of $75-85^{\circ} \mathrm{C}$ and under stirring using a small magnetic bar. After complete evaporation of the organic phase, the temperature and agitation were maintained for 30 min to eliminate any residual chloroform. In addition, the sample was centrifuged twice and the buffer $(5 \mathrm{mM}$ phosphate, $150 \mathrm{mM}$ $\mathrm{NaCl}$ and $0.2 \mathrm{mM}$ EDTA, $\mathrm{pH}$ 7.4) replaced. The sample volume was adjusted to $0.7 \mathrm{~mL}$ and extruded through a $0.8-\mu \mathrm{m}$ pore polycarbonate filter using an Avanti mini-extruder (Avanti Polar Lipids, Inc., Alabaster, AL) with the block heated to a temperature above the gel-liquid crystalline phase transition for each phosphatidylcholine. The extrusion process, consisting of around 20 serial extrusions, was repeated using polycarbonate filters with 0.4 and $0.2 \mu \mathrm{m}$ pores to obtain unilamellar vesicles of approximately $0.2 \mu \mathrm{m}$ in diameter. Finally, the vesicle suspension was transferred to a capillary, which was then flame-sealed. The quality and reproducibility of each preparation were monitored by the EPR spectrum of the spin probe, which is highly sensitive to the presence of any probe aggregates. Alternatively, the phospholipids DPPC and PC-18 were dissolved in a mixture of chloroform:methanol $(2: 1)$ and dried under a nitrogen stream. The residual solvent was removed by vacuum-drying the tube overnight. In the hydration step to form multilamellar vesicles, the samples were incubated in PBS for about $5 \mathrm{~min}$ at $60{ }^{\circ} \mathrm{C}$ and subsequently vortexed several times before the extrusion process. This method of preparation is the one most commonly reported in the literature, but was not suitable for the preparation of PC-20 and PC-22 vesicles. The latter method was used in the preparation of samples containing cholesterol, which was dissolved together with an appropriate amount of phospholipid.

\section{EPR spectroscopy}

EPR spectroscopy was carried out with a Bruker ESP 300 spectrometer (Bruker, Rheinstetten, Germany) equipped with an ER 4102 ST resonator and a Bruker temperature controller. The instrument settings were: microwave power of $2 \mathrm{~mW}$; modulation frequency of $100 \mathrm{kHz}$; modulation amplitude of $1.0 \mathrm{G}$; magnetic field scan of $100 \mathrm{G}$; sweep time of $168 \mathrm{~s}$; and a detector time constant of $41 \mathrm{~ms}$. The sample, in a sealed capillary, was introduced into a 3-mm I.D. quartz tube containing mineral oil and a fine-wire thermocouple to monitor the sample temperature. EPR spectral simulations were performed using the NLLS program developed by Jack H. Freed and coworkers. ${ }^{14,15}$ This program allows a single spectrum to be fitted with a model of two spectral components having different mobility and magnetic tensor parameters, yielding both the relative populations and the associated rotational diffusion rates. In the spectral calculations, the NLLS program includes the magnetic g- and A-tensors and the rotational diffusion tensor, $\mathrm{R}$, which are expressed in a system of Cartesian axes fixed in the spin-labeled molecule. To reduce the number of parameters and simplify the simulation, a spherical form was assumed for the $\mathrm{R}$ tensor (isotropic motion). Similarly to previous studies, ${ }^{16,17}$ the magnetic parameters were determined based on a global analysis of the overall spectra obtained in this work, after which all the EPR spectra were simulated using the same parameters. 
In this work, the spectra were simulated with a model of one or two spectral components. The component with greater rigidity and higher polarity was denoted as component 1 and the other as component 2. However, at temperatures above the gel-liquid crystalline phase transition, the magnetic parameters of component 1 underwent minor changes. Table $1 \mathrm{~S}$ of the supplementary materials, shows the magnetic parameters of these components.

The EPR spectra were also simulated in the fast motion regime, using the EPRSIM (version 4.99) software program. ${ }^{18,19}$ The input parameters for the magnetic interaction tensors $\mathrm{A}$ and $\mathrm{g}$, which were the same as those listed in Table $1 \mathrm{~S}$ (supplementary materials), were corrected linearly by this fitting program through polarity correction factors applied to the trace of A- and g-tensors, denoted by parameters $p_{A}$ and $p_{g}$, respectively. While NLLS generates the average rotational diffusion rate, Rbar, EPRSIM provides the parameter of rotational correlation time, $\tau_{\mathrm{c}}$, which follows the relationship $\tau_{\mathrm{c}}=1 / 6$ Rbar.

\section{RESULTS}

\section{Analysis of EPR spectra}

Two-component EPR spectra are very often found for lipid spin labels in membrane models. Figure 2 depicts spectra of several spin labels (Figure 1) in DPPC vesicles at three selected temperatures for each probe. Double arrows indicate the magnetic-field positions where the features indicative of the presence of two components are more clearly visible. Increasing the temperature caused the relative fractions of component 1 (the less mobile component) to decrease in the spectra. These EPR spectra evidencing two components appeared at higher temperatures for the stearic acid derivative spin labels than for methyl ester of stearic acid labels and at different temperatures for the chain-labeled PCs. For 5-, 12- and 16-DMS, the two components were more evident at around $30{ }^{\circ} \mathrm{C}$ while the relative populations

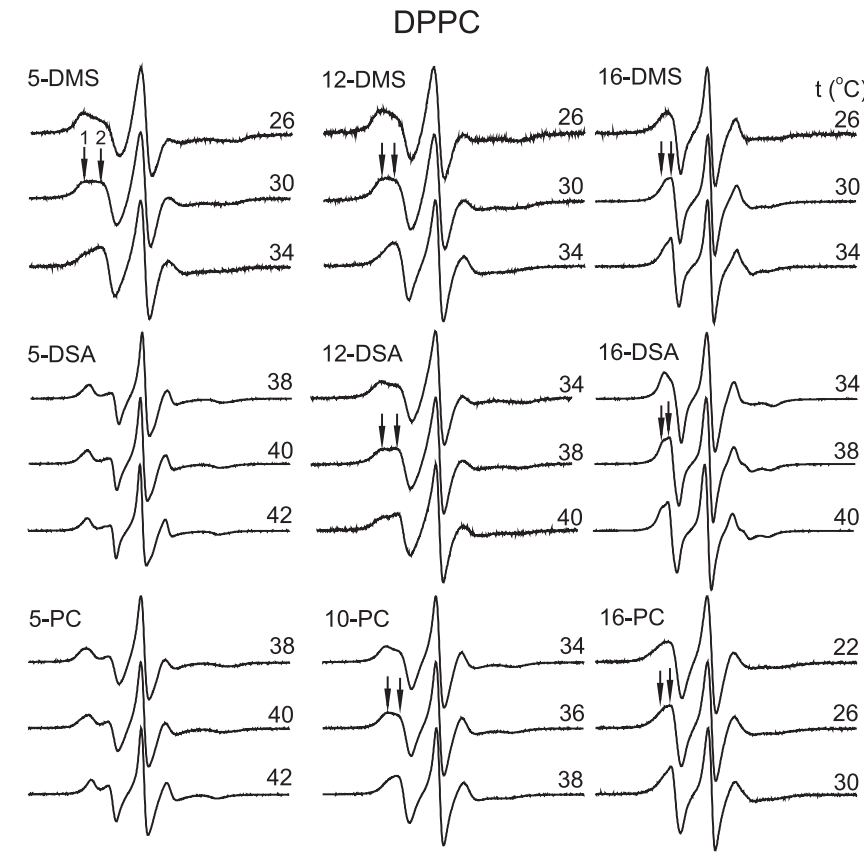

Figure 2. EPR spectra of spin label analogs of methyl ester of stearic acid (5- 12- and 16-DMS), stearic acid (5-, 12- and 16-DSA) and DPPC (5-, 10and 12-PC) in DPPC vesicles at temperatures indicated. The double arrows indicate the features in the spectrum where the coexistence of two spectral components is more clearly visible. In this work, the total scanning range of the magnetic field of all the spectra was $100 \mathrm{G}$ were essentially the same for the three spin labels at this temperature. This fact suggests that, for these spin probes, the nitroxide position in the acyl chain does not affect the population ratio between the two components. This good performance in terms of low influence of the nitroxide position was not the case with the other two probes. In the case of 5-DSA and 5-PC, strong interactions of nitroxide radical with the polar groups of the bilayer interface probably occurs, making their line shapes different to the other positional isomers and consequently more difficult to analyze. As the spectra of probes derived from methyl ester of stearic acid are simpler and well behaved, attention in this study was focused on 5-DMS.

Figure 3 shows several experimental and best-fit EPR spectra of 5-DMS in DPPC at temperatures below and above the phase transition $\left(\mathrm{T}_{\mathrm{m}}\right)$. Component 1 predominated in the spectra at temperatures between 2 and $18{ }^{\circ} \mathrm{C}$, and an appreciable fraction of component 2 (20-30\%) was visible at $30^{\circ} \mathrm{C}$. Two spectral components were again visible in the liquid-crystalline phase, and the fittings for this phase were performed with minor changes in the magnetic parameters of component 1 . As the EPRSIM fitting program performed well in the fast motion regime, all the results obtained with the NLLS for the liquid-crystalline phase were checked using this program.

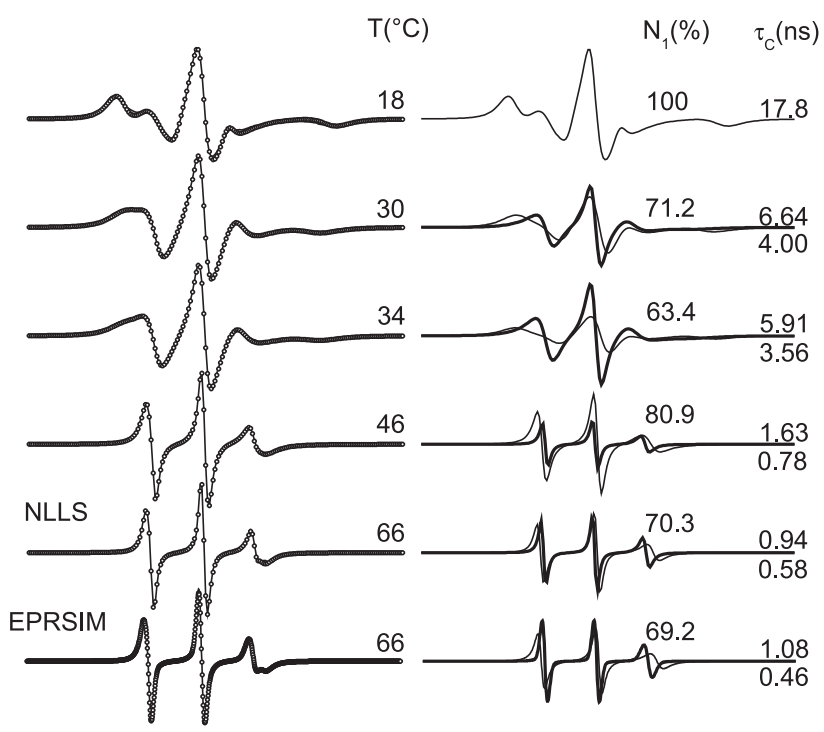

Figure 3. (Left column) Experimental (black lines) and best-fit (open circles) EPR spectra of 5-DMS in DPPC vesicles at temperatures indicated. (Right column) Best-fit spectral components 1 (thin lines) and 2 (thick lines) and best-fit parameters. The spectrum at $18^{\circ} \mathrm{C}$ was fitted with one component and the others with two components. The NLLS fitting program was used in this work, and the EPRSIM program was also used to confirm the results for spectra in the fast motion regime. $N_{1}$ is the fraction of component 1 in the spectrum and $\tau_{c}$ is the rotational correlation time

As Figure 4 indicates, DPPC in the liquid-crystalline phase exhibits two components of the spin labels 5- and 7-DMS, but only one for the 12-DMS. The degree of penetration of water molecules into the hydrophobic interior can be deduced from polarity profile measurements using membrane models. The EPR parameter - isotropic ${ }^{14} \mathrm{~N}$-hyperfine coupling constant, $\mathrm{a}_{0}$, increases with increasing dielectric constant or polarity of the solvent in which the nitroxide radical is dissolved. It has been shown that spin-labeled glycerophospholipids with the doxyl-nitroxide group at position $n$ in the $s n$ - 2 chain ( $n$-PCSL) in DPPC membranes have $\mathrm{a}_{0}$ values ranging from $15.0 \mathrm{G}$ for $n=4$ to $14.5 \mathrm{G}$ for $n=16$, with an abrupt drop in polarity between $n=7$ and $n=9 .{ }^{20}$ As indicated in Figure 4, the EPR spectrum of 5-DMS can be simulated with two components of different polarity (Table $1 \mathrm{~S}$ ) and mobility. 
This suggests that EPR spectroscopy identifies the two components by associating lower mobility with higher polarity (component 1) and higher mobility with lower polarity (component 2 ). This can be understood by imagining a transverse fluctuation of 5-DMS between shallow and deep locations in the membrane. Whenever the spin label goes to the deepest region of the membrane, whose environment is characterized by lower polarity and higher mobility, it contributes to component 2, and thus two spin label populations are visible. Since there is less probability of 7-DMS reaching the region of higher polarity, its population in component 1 is also smaller. Similarly, the fact that 12-DMS has only one component suggests that most of the nitroxide moieties of this spin probe do not reach the region of higher polarity in the membrane. Figure $1 \mathrm{~S}$ of the supplementary materials shows the experimental and best-fit EPR spectra of the other PCs analyzed in this work. Note that, at $42^{\circ} \mathrm{C}$, the spectrum tends to assume the line shape of a single component for all the PCs, indicating that, at this temperature, the difference in mobility between the two components is already diminished.

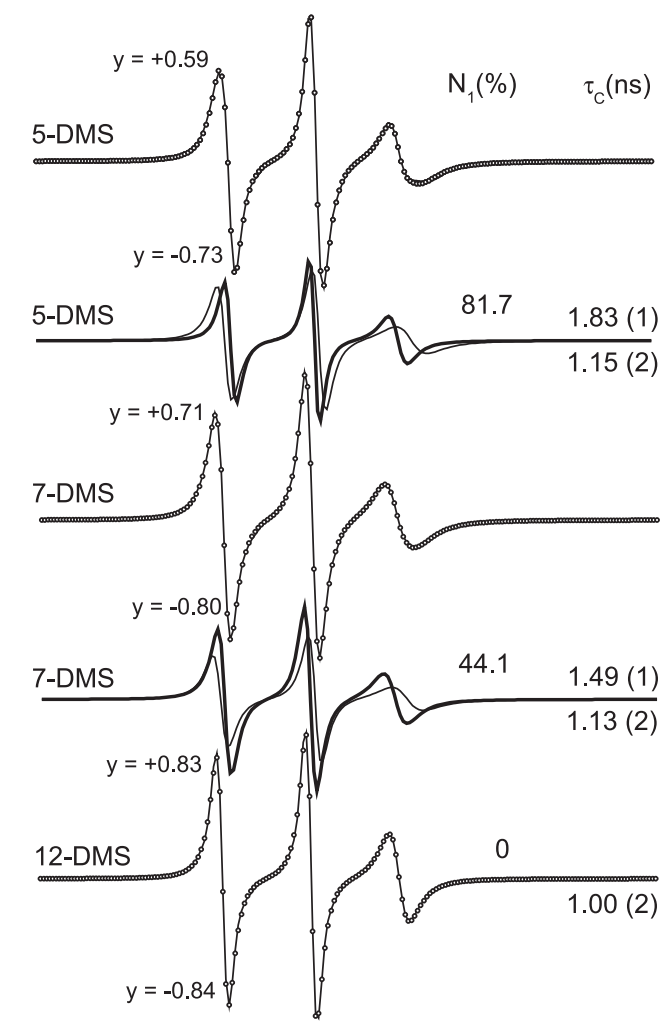

Figure 4. Experimental (black lines) and best-fit (open circles) EPR spectra of 5-DMS (a), 7-DMS (c) and 12-DMS (e) in DPPC vesicles at $44^{\circ} \mathrm{C}$. The best-fit spectral components 1 and 2 are shown in thin and thick lines for the spin labels 5-DMS (b) and 7-DMS (d), respectively. The spectrum of 12-DMS can be simulated with one component. The peak heights above and below the baseline on the first resonance line of spectra $(a),(c)$ and $(d)$ are indicated by the $y$-values. Note, from these values, that the first resonance line is symmetric for 12-DMS and more asymmetric for 5-DMS than for 7-DMS. This asymmetry is indicative of the presence of two components. The rotational correlation time, $\tau_{O}$ and percentage of component $1, N_{l}$, are lower for spin labels with the doxyl group located further along the acyl chain

\section{Spin label dynamics and populations in the two main locations of the lipid bilayer}

The temperature dependence of rotational correlation time and percentage of relative population of 5-DMS in component 1 is shown in Figure 5 for the PCs in this study. Below $26^{\circ} \mathrm{C}$, the spectra were fitted with a single component. At $30^{\circ} \mathrm{C}$, the rotational correlation time of component 2 was about half that of component 1 . In the samples PC-18 to PC-22, whose phase-transition temperatures are above $50{ }^{\circ} \mathrm{C}$, the mobility of the two components converged at 50 ${ }^{\circ} \mathrm{C}$ and the spectral resolution was insufficient to identify the two components; hence, the spectra were fitted with a single component for these temperatures. A pronounced increase in the population of component 2 in DPPC was detected when the temperature approached the phase transition. It is remarkable that the dynamics of 5-DMS in all the PCs was similar at temperatures in the $2-30{ }^{\circ} \mathrm{C}$ range. The EPR parameter - outer hyperfine splitting, $2 \mathrm{~A}_{\max }$, which is very sensitive to the probe's mobility and can be measured directly in the spectra of a single component, was used to confirm that, up to $22^{\circ} \mathrm{C}$, the measurements shown in Figure 5A did not differ significantly in mobility. The measurement of $2 \mathrm{~A}_{\max }$ at $2{ }^{\circ} \mathrm{C}$ in all the PCs was $62.5 \mathrm{G}$, with an experimental error of $0.5 \mathrm{G}$ (data not shown). The spectra of all the lipids were also very similar at $78^{\circ} \mathrm{C}$. However, significant
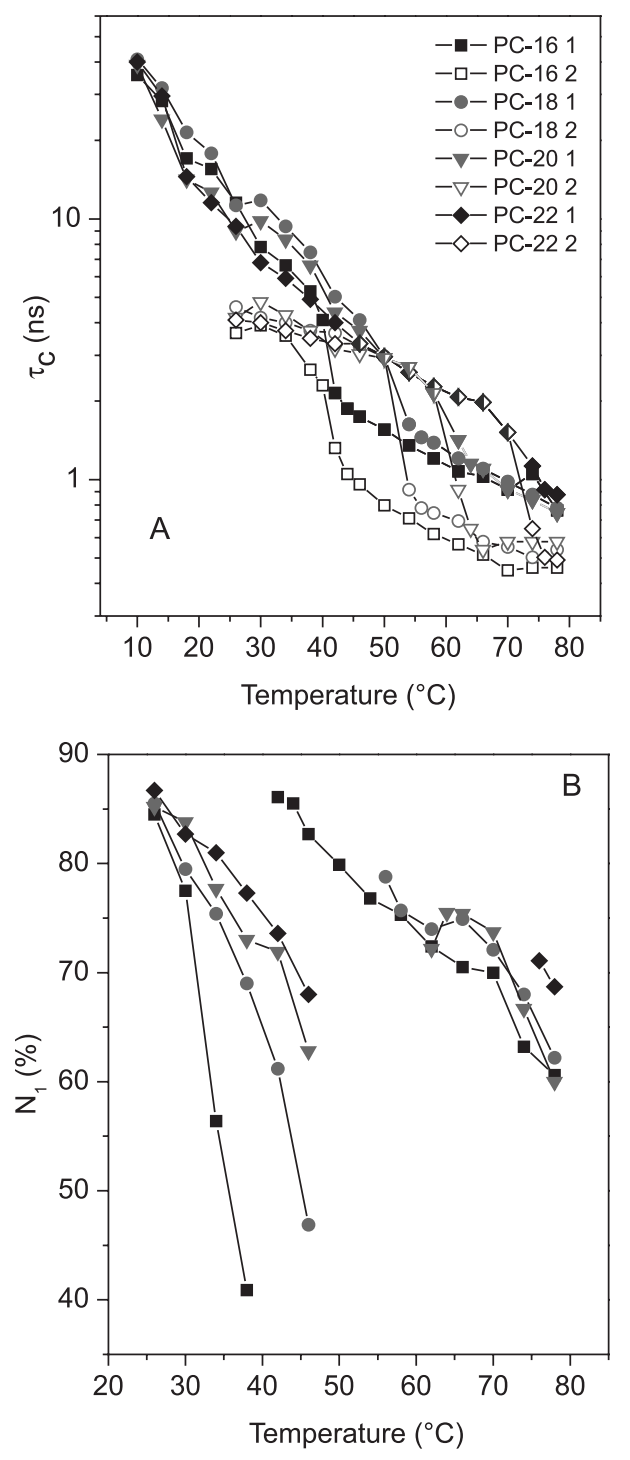

Figure 5. (A) Rotational correlation time, $\tau_{c}$ of 5-DMS in phosphatidylcholine vesicles as a function of temperature. The best-fit spectra in this study were obtained by NLLS fitting, using a simulation model with one or two spectral components. (B) Percentage of components $1, N_{1}$, in the spectra composed of components 1 and 2, versus temperature. The closed and open symbols refer to components 1 and 2, respectively 
changes in both mobility and spin-probe populations were detected at temperatures near $\mathrm{T}_{\mathrm{m}}$.

Since the two spectral components are in thermodynamic equilibrium and the fitting program provides the relative populations of spin label in components $1, \mathrm{~N}_{1}$, and $2, \mathrm{~N}_{2}$, the equilibrium constant, $\mathrm{K}$, between these two components $\left(\mathrm{K}=\mathrm{N}_{2} / \mathrm{N}_{1}\right)$ can be determined. The standard Gibbs free energy change required to transfer the spin label from component 1 to $2, \Delta \mathrm{G}^{\circ}{ }_{1 \rightarrow 2}$, was calculated as follows: ${ }^{21}$

or

$$
\Delta \mathrm{G}^{\mathrm{o}}=-\mathrm{RT} \ln \mathrm{K},
$$

$$
\ln \mathrm{K}=-\Delta \mathrm{H}^{\mathrm{o}} / \mathrm{RT}+\Delta \mathrm{S}^{\mathrm{o}} / \mathrm{R}
$$

where $\mathrm{R}$ is the gas constant, $\mathrm{T}$ is the absolute temperature, $\Delta \mathrm{H}^{\circ}$ is the standard enthalpy change to transfer the spin probe from component 1 to 2 , and $\Delta \mathrm{S}^{\circ}$ is the associated entropy change. These thermodynamic parameters can be obtained from a van't Hoff plot of $\operatorname{lnK}$ versus $1 / \mathrm{T}$, as shown in Figure 6. In this type of plot, the slope coefficient of each curve yields $\Delta \mathrm{H}^{\circ} / \mathrm{R}$ whereas the corresponding intercept yields $\Delta \mathrm{S} / \mathrm{R}$. The $\Delta \mathrm{H}^{\circ}$ can be understood as the apparent energy needed to dissociate the motionally restricted nitroxide moiety from the polar interface of the bilayer through an endothermic process with increased entropy. Table 1 lists the calculated values of the apparent thermodynamic parameters. In the liquid-crystalline phase, the van't Hoff plot for DPPC appears linear in the temperature range of 42 to $78^{\circ} \mathrm{C}$, suggesting that enthalpy and entropy changes are constant. The thermodynamic parameters of the other lipids were not calculated, but it can be inferred from the plots that these were similar to DPPC. In the gel state, the fitting program only distinguishes the two components up to $46^{\circ} \mathrm{C}$ for the PCs with $\mathrm{T}_{\mathrm{m}}$ above $50{ }^{\circ} \mathrm{C}$, and in the case of DPPC, the proximity to the melting temperature anticipates the transfer of the spin probe to component 2 , thereby providing higher values of enthalpy and entropy change.

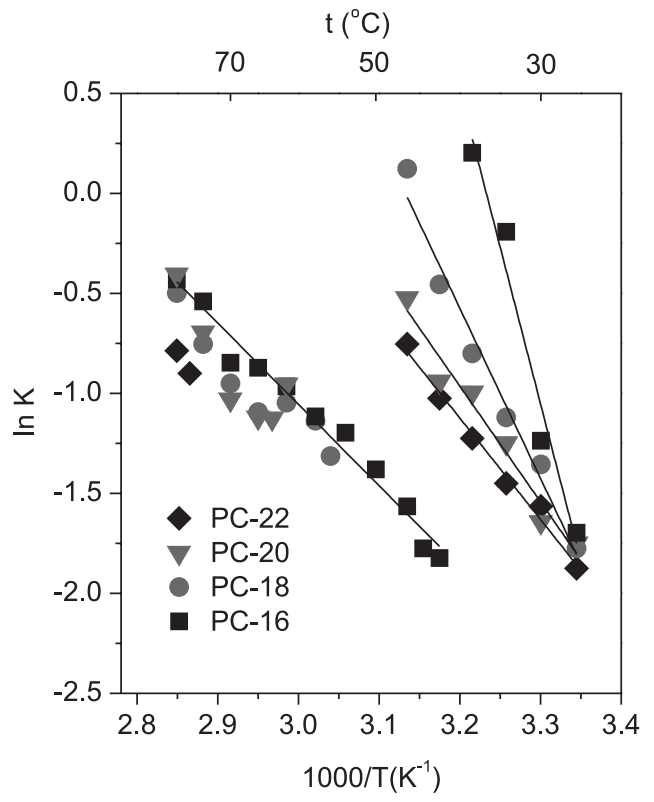

Figure 6. van't Hoff plot. Natural logarithm of $K$, the population ratio between components 2 and 1, $N_{2} / N_{1}$, of 5-DMS in phosphatidylcholine vesicles versus the reciprocal absolute temperature

Another EPR-fitting program (EPRSIM) also performed well in simulating the spectra of these PCs in the liquid-crystalline phase. This program has the advantage of making minor corrections to the mean values of magnetic g- and A-tensors (Table 1S), avoiding
Table 1. Changes in standard Gibbs free energy $\left(\right.$ at $\left.34^{\circ} \mathrm{C}\right)$, enthalpy and entropy changes accompanying the transference of spin label 5-DMS from component 1 to 2 in vesicles of phosphatidylcholines (PC-16 to PC-22) below the main phase transition and after the transition for PC-16

\begin{tabular}{lccc}
\hline Samples & $\begin{array}{c}\Delta \mathrm{G}^{\circ}{ }_{1 \rightarrow 2} \\
\mathrm{kcal} \mathrm{mol}^{-1}\end{array}$ & $\begin{array}{c}\Delta \mathrm{H}^{\mathrm{o}}{ }_{1 \rightarrow 2} \\
\mathrm{kcal} \mathrm{mol}^{-1}\end{array}$ & $\begin{array}{c}\Delta \mathrm{S}^{\circ}{ }{ }^{\rightarrow 2} \\
\mathrm{cal} \mathrm{mol}^{-1} \mathrm{~K}^{-1}\end{array}$ \\
\hline PC-16 & 0.2 & 31.0 & 100.2 \\
PC-18 & 0.6 & 16.8 & 52.7 \\
PC-20 & 0.7 & 11.4 & 34.7 \\
PC-22 & 0.8 & 10.0 & 29.9 \\
PC-16 after transition & 1.2 & 8.0 & 22.1 \\
PC-16 + cholesterol $^{\mathrm{a}}$ & 0.5 & 2.8 & 7.6 \\
\hline
\end{tabular}

The numerical values were calculated based on eqs. (1) and (2), using the data from the van't Hoff graph (Fig. 6). ${ }^{a}$ DPPC with $40 \mathrm{~mol} \%$ cholesterol $\left(26-78{ }^{\circ} \mathrm{C}\right)$

possible inaccuracies in the chosen input data. Figure $2 \mathrm{~S}$ of the supplementary materials compares the results obtained with the two fitting programs for the rotational correlation time and distribution of 5-DMS in DPPC. Interestingly, the two fitting programs yielded similar results, providing important confirmation of the thermodynamic parameters (Table 1) determined for the fluid phase.

\section{Effect of cholesterol on 5-DMS dynamics and distribution in the bilayer}

The temperature dependencies of $\tau_{\mathrm{C}}$ and $\mathrm{N}_{1}$ of 5-DMS in DPPC and DPPC containing $40 \mathrm{~mol} \%$ cholesterol are shown in Figure $3 \mathrm{~S}$ (supplementary materials). Below $41^{\circ} \mathrm{C}$, the two components did not differ significantly in mobility, but showed differences in their relative populations. However, above $41{ }^{\circ} \mathrm{C}$, the samples' mobility and relative populations differed considerably. The data in Figure $3 \mathrm{~S}$ are representative of three sample preparations containing different molar ratios of cholesterol $(30,35$ and $40 \%)$, which presented very similar results. The sample with cholesterol showed a gradual reduction in $\tau_{\mathrm{C}}$-values with increasing temperature, and in the case of component 1 , there was a slight change in the slope at around $40^{\circ} \mathrm{C}$. On the other hand, the abrupt change in the $\mathrm{N}_{1}$ plot for the control sample at $\mathrm{T}_{\mathrm{m}}$ was reduced to a minor fluctuation for the sample with cholesterol.

\section{DISCUSSION}

In the current work, a detailed study of the thermal behavior of spin label 5-DMS in phosphatidylcholine vesicles indicated that when the membrane's phase-transition temperature exceeds $50{ }^{\circ} \mathrm{C}$, the mobility of the two spectral components converges to similar values and cannot be separated in the temperature interval from $50{ }^{\circ} \mathrm{C}$ up to the membrane's melting temperature. This indicates that the interactions responsible for the restricted motion of a probe fraction are reduced at $50{ }^{\circ} \mathrm{C}$. However, in the case of the DPPC membrane with a $\mathrm{T}_{\mathrm{m}}$ of $41^{\circ} \mathrm{C}$, significant changes were observed in the dynamics and distribution of the probe as the temperature approached the phase transition (Figure 5), which were also reflected in the calculated values of the apparent $\Delta \mathrm{H}^{\circ}$ and $\Delta \mathrm{S}^{\circ}$ (Table 1). This probe's instability is consistent with membrane reorganizations that occur at temperatures near $\mathrm{T}_{\mathrm{m}}$ and is probably the cause for the increased permeability of phospholipid bilayers to small water-soluble molecules in the regions of two-phase coexistence. ${ }^{22}$

Two-component EPR spectra of the cholestane spin probe (cholesterol analog) have been observed in well-aligned multilayers containing a mixture of the lipids 1-palmitoyl-2-oleoyl-sn-glycero-3-phosphocholine, 
cholesterol, and dioctanoylglycerol, which are visible both with the external magnetic field parallel and perpendicular to the bilayer plane. ${ }^{15}$ Although the spectra for the two magnetic-field orientations were very different at $36^{\circ} \mathrm{C}$, the authors reported that the relative populations of the two spectral components in each spectrum were essentially the same and appeared to vary almost reversibly according to temperature and membrane composition. However, the origin of these two spectral components in single phospholipid bilayers remains poorly understood and has generally been attributed to heterogeneity of the membrane. Several spin labels have previously been examined by EPR spectroscopy to study the lipid dynamics of stratum corneum, in a bid to understand why skin permeability is reduced by dehydration of this membrane. ${ }^{23,24}$ Upon decreasing the hydration level of stratum corneum, a gradual decrease in motional dynamics was detected and, most importantly, upon dehydration the population of the second spectral component for various spin labels was diminished, tending to disappear in response to total dehydration of the stratum corneum. Similar results have been observed for phospholipid vesicles (data not shown). These experimental data suggest that the more rigid component could be assigned to the spin labels that are hydrogen-bonded to the polar interface, directly or mediated by water molecules (rigid structure). Further, the more mobile component may arise when the polar group of the probe within the membrane is H-bonded to free water molecules (mobile structure) or non-H-bonded. However, even assuming that $\mathrm{H}$-bonding interactions are the key point for the resolution of two spectral components, there are clearly other interactions arising from hydrophobic and van der Waals forces. These interactions are also involved in the set of intermolecular interactions that define the two distinct probe environments within the membrane and certainly contribute to the values of the apparent enthalpy and entropy changes observed here (Table 1).

DPPC bilayers containing $40 \mathrm{~mol} \%$ cholesterol form the liquid-ordered phase characterized by several changes, especially in the temperature range of the fluid phase for pure DPPC. ${ }^{25,26}$ The main changes are: better packing of phospholipids and their orientational order, increased thickness of the hydrophobic layer and reduction of average cross-sectional area per molecule. ${ }^{25}$ Our results with the spin label 5-DMS indicated that at temperatures below $41{ }^{\circ} \mathrm{C}$, cholesterol at $40 \%$ molar did not significantly affect the lipid mobility. It is noteworthy that the outer hyperfine splitting, $2 \mathrm{~A}_{\max }$, increased by about $1 \mathrm{G}$, which could indicate a slight stiffening of the bilayer containing cholesterol. However, a more accurate analysis with the NLLS fitting program indicated that this was an artifact and that cholesterol does, in fact, increase the polarity of the bilayer, since the best fitting of EPR spectra at lower temperatures $\left(2-10^{\circ} \mathrm{C}\right)$ was found with a slightly larger magnetic parameter $A_{z z}$ (Table 1S). This result is in agreement with reported measurements of the EPR parameter isotropic ${ }^{14} \mathrm{~N}$-hyperfine coupling constant, $\mathrm{a}_{0}$, for DPPC, where equimolar cholesterol increased the $\mathrm{a}_{0}$-value by $0.3 \mathrm{G}$ for the nitroxide at position C-5 of the chain (the case of 5-DMS). ${ }^{20}$ In the presence of $40 \mathrm{~mol} \%$ cholesterol, the abrupt increase in the dynamics of 5-DMS that occurs at $41{ }^{\circ} \mathrm{C}$ with pure DPPC was not observed, and above this temperature the dynamics of the probe were considerably diminished. It is interesting to compare our results with data of the $2 \mathrm{~A}_{\max }$ parameter reported for sphingomyelin containing $40 \mathrm{~mol} \%$ cholesterol, which were measured with spin label analogs of phosphatidylcholine and sphingomyelin labeled in the $\mathrm{C}-5$ position in the chain. ${ }^{27}$ At $22{ }^{\circ} \mathrm{C}$, which corresponds to the gel phase of pure sphingomyelin bilayers, the reported values of $2 \mathrm{~A}_{\max }$ were similar to those obtained for 5-DMS in DPPC plus cholesterol, and at $50{ }^{\circ} \mathrm{C}$ the pronounced increase in $2 \mathrm{~A}_{\max }$ caused by cholesterol was consistent with the increase in the rotational correlation time observed here.
The better packing of phospholipids caused by cholesterol above $41{ }^{\circ} \mathrm{C}$ induced a relocation of 5-DMS towards the second component (Figure 3S). The results of the present work are in agreement with studies by fluorescence spectroscopy, which have also shown that several membrane probes may occupy both shallow and deep locations in the bilayer. ${ }^{3-7}$ A study with the fluorescent probe 4'-dimethylamino-3-hydroxyflavone in bilayer vesicles of EYPC has shown that the addition of $30 \mathrm{~mol} \%$ cholesterol induced strong changes in the concentrations of the probe in H-bonded and H-bond free forms, suggesting redistribution of the dye from the membrane surface to the hydrophobic region of the bilayer. ${ }^{5}$ Moreover, similar relocations of the probe Prodan caused by cholesterol were also observed in model membranes of phosphatidylcholines.$^{28}$ We believe that the study of the bimodal distribution of molecules into membranes, resulting in the resolved two-component EPR spectra, may make important contributions toward a better understanding of the permeation of molecules in cell membranes. It would be interesting to verify whether the spin labels with the largest formations of the second spectral component also have higher rates of flip-flop. The proposed transverse fluctuation of lipids between two main locations in the membrane is consistent with some structurally relevant mechanisms for the antioxidant activity of tocopherol. To exert its role as an antioxidant, tocopherol must have the ability to access both peroxidising unsaturated lipids in bilayer membranes and the aqueous reductant ascorbate ${ }^{29}$. Possible depths that the chromanol portion of $\alpha$-tocopherol may occupy in phospholipid bilayers have been investigated using several techniques in terms of the occurrence of a hydrogen bond between the phenol and the polar groups of the phospholipids. ${ }^{29}$

\section{CONCLUSION}

This study examined the hypothesis of two main accommodations of spin-labeled lipids in simple bilayers. The fitting program NLLS and simulation models of one or two components were used to monitor the dynamics and relative populations in each component. Based on the $\Delta \mathrm{H}^{\circ}$ values, it was also found that above the phase transition temperature, the ability of the probe to move towards the second component was independent of the acyl chain length of the phosphatidylcholines, but in the gel phase this transfer of the probe was accompanied by major changes in $\Delta \mathrm{H}^{\circ}$ and $\Delta \mathrm{S}^{\circ}$ for short-chain phospholipids. Two-component EPR spectra appear more often for spin labels that interact more weakly with polar groups of the bilayer, as is the case of 5-DMS, whose polar groups are only carbonyl and nitroxyl radical. At gel-fluid phase transition, the rotational correlation time and relative population of these components for 5-DMS in DPPC undergo abrupt changes which are completely attenuated in the presence of $40 \mathrm{~mol} \%$ cholesterol. Moreover, above $41^{\circ} \mathrm{C}$, cholesterol favored the formation of the second component, but in the presence of cholesterol the mobility of spin labels in this component was decreased. Also, the calculated enthalpy change to transfer the 5-DMS from component 1 to 2 was smaller for the sample containing cholesterol. Our current interpretation is that EPR spectroscopy identifies the two components, associating lower mobility with higher polarity and higher mobility with lower polarity. This could be understood by imagining a transverse fluctuation of 5-DMS between shallow and deep locations in the membrane. However, the origin of the formation of two-component EPR spectra remains poorly understood and to reach a more reliable interpretation further studies are warranted not only by EPR spectroscopy but also employing other techniques.

\section{SUPPLEMENTARY MATERIAL}

Available at http://quimicanova.sbq.org.br in the form of a PDF 
file with free access, comprising Table $1 \mathrm{~S}$ and Figures $1 \mathrm{~S}, 2 \mathrm{~S}$ and $3 \mathrm{~S}$ cited in the text.

\section{ACKNOWLEDGMENTS}

The authors are indebted to the Brazilian research funding agencies CNPq, CAPES and FAPEG. A. Alonso is grateful to CNPq for a research grant.

\section{REFERENCES}

1. Jost, P.C.; Griffith, O.H.; Capaldi, R.A.; Vanderkooi, G.; Proc. Natl. Acad. Sci. U.S.A. 1973, 70, 480.

2. Griffith, O.H.; Jost, P.C.; Lipid spin labels in biological membranes, In Spin Labeling Theory and Applications; Berliner, L.J., ed.; Academic Press: New York, 1976.

3. Asuncion-Punzalan, E.; Kachel, K.; London, E.; Biochemistry 1998, 37, 4603.

4. Kaiser, R.D.; London, E.; Biochim. et Biophys. Acta 1998, 1375, 13.

5. Klymchenko, A.S.; Duportail, G.; Demchenko, A.P.; Mély, Y.; Biophys. J. 2004, 86, 2929.

6. Chong, P.L.; Biochemistry 1988, 27, 399.

7. Doroshenko, A.O.; Sychevskaya, L.B.; Grygorovych, A.V.; Pivovarenko, V.G.; J. Fluorescence 2002, 12, 455.

8. De Queirós, W.P.; De Sousa Neto, D.; Alonso, A.; J. Controlled Release 2005, 106, 374.

9. Dos Anjos, J.L.V.; Neto, D.D.; Alonso, A.; European J. Pharmaceutics and Biopharmaceutics 2007, 67, 406.

10. Anjos, J.L.Vd.; Neto, D.dS.; Alonso, A.; Int. J. Pharm. 2007, 345, 81.

11. Alonso, L.; Mendanha, S.A.; Marquezin, C.A.; Berardi, M.; Ito, A.S.; Acuña, A.U.; Alonso, A.; Int. J. Pharm. 2012, 434, 391.

12. Desoti, V.C.; Lazarin-Bidóia, D.; Sudatti, D.B.; Pereira, R.C.; Alonso, A.; Ueda-Nakamura, T.; Dias Filho, B.P.; Nakamura, C.V.; de Oliveira Silva, S.; Mar. Drugs 2012, 10, 1631.
13. Mendanha, S.A.; Moura, S.S., Anjos, J.L.; Valadares, M.C.; Alonso, A.; Toxicol. In Vitro 2013, 27, 323.

14. Schneider, D.J.; Freed, J.H.; Calculating slow motional magnetic resonance spectra: a user's guide, In Biological Magnetic Resonance, vol. 8., Berliner, L.J.; Reuben J., eds.; Plenum Press: New York, 1989.

15. Budil, D.E.; Lee, S.; Saxena, S.; Freed, J.H.; J. Magn. Reson. A 1996, $120,155$.

16. Alonso, A.; Dos Santos, W.P.; Leonor, S.J., Tabak, M.; Biophys. J. 2001, $81,3566$.

17. Alonso, A.; Da Silva, J.V.; Tabak, M.; Biochim. et Biophys. Acta 2003, 1646, 32.

18. Arsov, Z.; Schara, M.; Strancar, J.; J. Magn. Reson. 2002, 157, 52.

19. Stopar, D.; Strancar, J.; Spruijt, R.B.; Hemminga, M.A.; Biophys. J. 2006, $91,3341$.

20. Marsh, D.; Proc. Natl. Acad. Sci. U.S.A. 2001, 98, 7777.

21. Van Holde, K.E.; Johnson, W.C.; Ho, P.S.; Principles of Physical Biochemistry, Prentice-Hall/PTR: New Jersey, 1998.

22. Clerc, S.G.; Thompson, T.E.; Biophys. J. 1995, 68, 2333.

23. Alonso, A.; Meirelles, N.C.; Tabak, M.; Biochim. Biophys. Acta 1995 , 1237, 6 .

24. Alonso, A.; Meirelles, N.C.; Yushmanov, V.E.; Tabak, M.; J. Invest. Dermatol. 1996, 106, 1058.

25. lpsen, J.H.; Mouritsen, O.G.; Bloom, M.; Biophys. J. 1990, 57, 405.

26. Sankaram, M.B.; Thompson, T.E.; Proc. Natl. Acad. Sci. U.S.A. 1991, $88,8686$.

27. Collado, M.I.; Goñi, F.M.; Alonso, A.; Marsh, D.; Biochemistry 2005, 44, 4911.

28. Bondar, O.P.; Rowe, E.S.; Biophys. J. 1999, 76, 956.

29. Atkinson, J.; Epand, R.F.; Epand, R.M.; Free Radic. Biol. Med. 2008, 44,739 . 
ELECTRON PARAMAGNETIC RESONANCE (EPR) SPECTRAL COMPONENTS OF SPIN-LABELED LIPIDS IN SATURATED PHOSPHOLIPID BILAYERS. EFFECT OF CHOLESTEROL

Heverton Silva Camargos ${ }^{\#}$ and Antonio Alonso*

Instituto de Física, Universidade Federal de Goiás, 74001-970 Goiânia - GO, Brasil

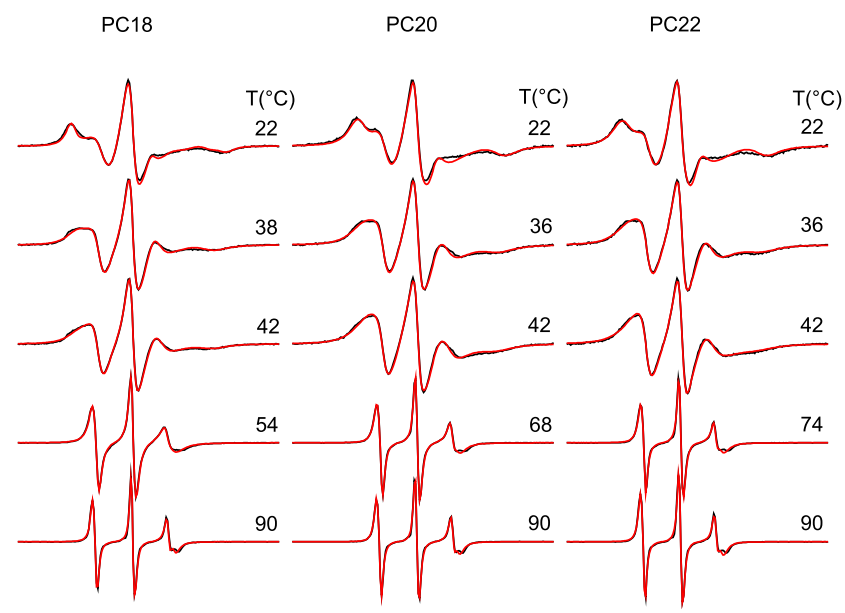

Figure 1S. Experimental (black line) and best-fit (red line) EPR spectra of 5-DMS in PC-18, PC-20 and PC-22 at temperatures indicated. The best-fit spectra were obtained by NLLS fitting, using a simulation model with one or two spectral components
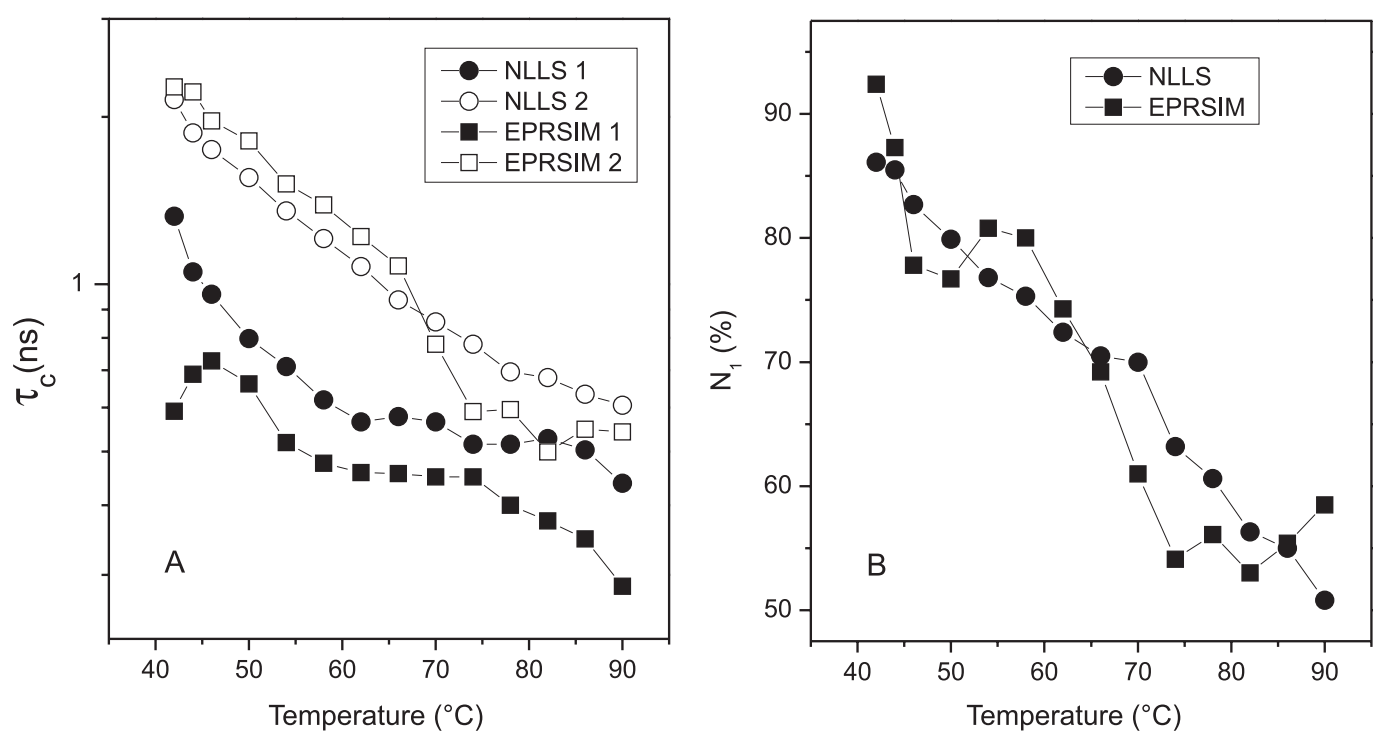

Figure 2S. Comparison of results obtained with the NLLS and EPRSIM fitting programs for the temperature dependence of the rotational correlation time, $t_{c}$ and relative population of component $1, N_{1}$, in the EPR spectra of 5-DMS in DPPC 

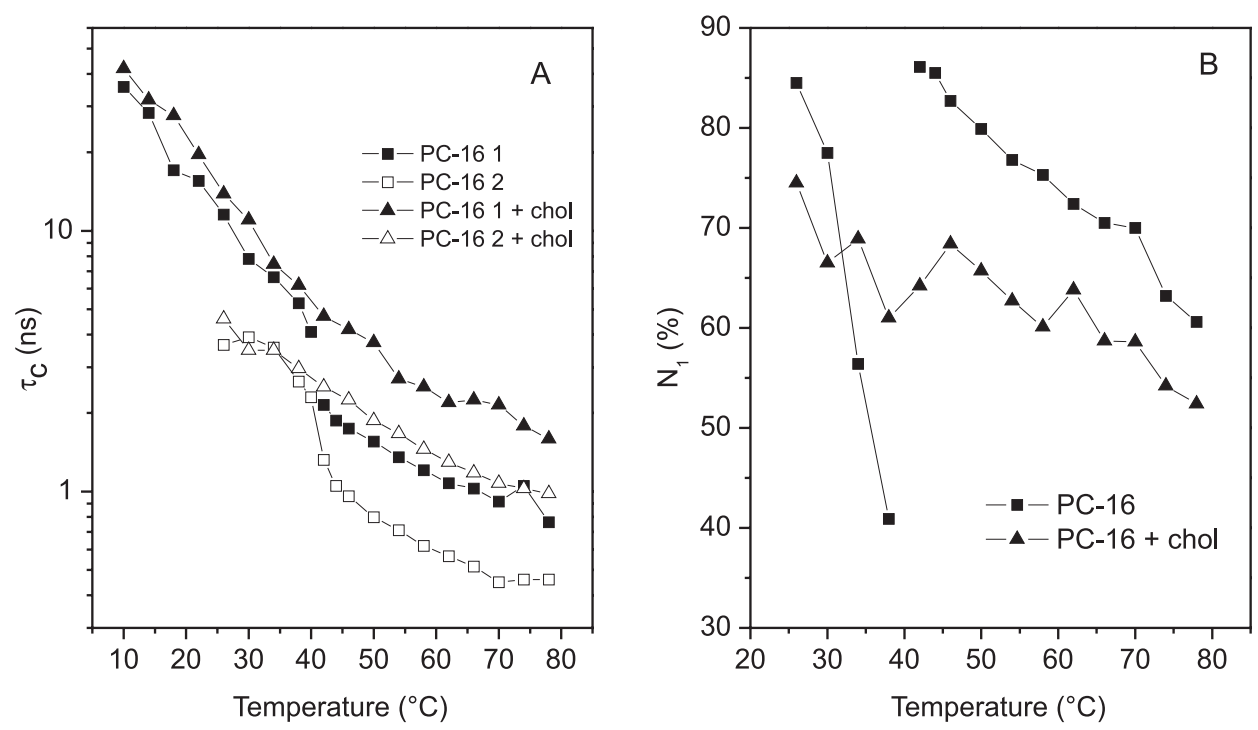

Figure 3S. Temperature dependencies of best-fit parameters of 5-DMS in DPPC and DPPC with $40 \mathrm{~mol} \%$ cholesterol: (A) rotational correlation time, $t_{c}$, and (B) percentage of component $1, N_{l}$

Table 1S. Principal components of the magnetic tensors $\mathrm{g}$ (g-factor) and A (hyperfine splitting) used in the nonlinear least-squares fitting program (NLLS)

\begin{tabular}{|c|c|c|c|}
\hline \multirow{2}{*}{ Parameter } & \multicolumn{2}{|c|}{ Component 1} & \multirow{2}{*}{ Component 2} \\
\hline & below $\mathrm{T}_{\mathrm{m}}$ & above $T_{m}$ & \\
\hline$g_{\mathrm{xx}}$ & 2.0080 & 2.0080 & 2.0082 \\
\hline $\mathrm{g}_{\mathrm{yy}}$ & 2.0060 & 2.0060 & 2.0062 \\
\hline $\mathrm{g}_{z z}$ & 2.0020 & 2.0020 & 2.0027 \\
\hline$A_{x x}(G)$ & 6.9 & 5.5 & 5.8 \\
\hline$A_{y y}(G)$ & 6.0 & 5.4 & 5.7 \\
\hline $\mathrm{A}_{z z}(\mathrm{G})$ & $31.6^{\mathrm{a}}$ & 34.1 & 31.6 \\
\hline$a_{0}(G)^{b}$ & 14.8 & 15.0 & 14.4 \\
\hline
\end{tabular}

${ }^{\mathrm{a}}$ For samples containing $40 \mathrm{~mol} \%$ cholesterol the $\mathrm{A}_{\mathrm{zz}}$ used was of $32.5 \mathrm{G} .{ }^{\mathrm{b}} \mathrm{a}_{0}=1 / 3\left(\mathrm{~A}_{\mathrm{xx}}+\mathrm{A}_{\mathrm{yy}}+\mathrm{A}_{\mathrm{zz}}\right)$. 